留- Revista Geográfica Digital. I GUNNE. Facultad de Humanidades. UNNE. Año 11. № 22. Julio - Diciembre 2014.

ISSN 1668-5180 Resistencia, Chaco

ler Congreso de la

Asociación Argentina de Sociología

"Nuevos Protagonistas en el contexto de América Latina y el Caribe"

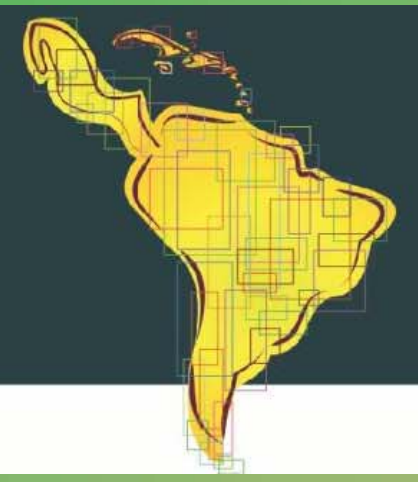

\title{
DESARROLLO RURAL TERRITORIAL Y
} TRANSFORMACIONESTERRITORIALES.

\section{Proyectos ¿para las igualdades o desigualdades?}

\author{
Prof. Mgter. Viviana C. PERTILE
}

Instituto de Geografía. Fac. de Humanidades. UNNE

Publicado en formato digital: Mgter. Prof. Viviana C. PERTILE. DESARROLLO RURAL TERRITORIAL Y TRANSFORMACIONES TERRITORIALES. Proyectos ¿para las igualdades o desigualdades? . Resúmenes. Revista Geográfica Digital. IGUNNE. Facultad de Humanidades. UNNE. Año 11. No 22. Julio - Diciembre 2014. ISSN 1668-5180 . Resistencia, Chaco. En: http://hum.unne.edu.ar/revistas/geoweb/default.htm 
Revista Geográfica Digital. I GUNNE. Facultad de Humanidades. UNNE. Año 11. № 22. J ulio - Diciembre 2014. ISSN 1668-5180 Resistencia, Chaco

Esta presentación forma parte de la exposición resumida de un trabajo publicado en el "I CONGRESO DE LA ASOCIACION ARGENTINA DE SOCIOLOGIA. Nuevos protagonistas en el contexto de América Latina y el Caribe". PRE ALAS Chaco, Encuentro Preparatorio Congreso ALAS Costa Rica 2015. Mesa 15. Territorio: desigual, fragmentado, excluido y vulnerable como consecuencia de las nuevas formas de producción en el marco de la globalización. Universidad Nacional del Nordeste. Campus Resistencia. Chaco, 29, 30 y 31 de octubre de 2014

Objetivo: Divulgar - en forma breve- en la comunidad académica y en la sociedad en general, trabajos realizados y expuestos en eventos para especialistas, que de otra forma no llegarán a diferentes interesados en estos temas, en especial a los que no han podido asistir a estos eventos.

Titulo: DESARROLLO RURAL TERRITORIAL Y TRANSFORMACIONES TERRITORIALES. Proyectos ¿para las igualdades o desigualdades?

Autorles: Viviana C. PERTILE. Instituto y Departamento de Geografía. Facultad de Humanidades. UNNE
e-mail:
/ vpertile@hum.unne.edu.ar

\section{Resumen}

La cuestión rural en la Argentina lleva consigo una discusión intensa, prolongada y sin resolver. La persistencia de la pobreza, la creciente desigualdad y el despoblamiento de amplias zonas rurales de nuestro país y de la provincia del Chaco, hablan de la poca efectividad que tuvieron las políticas públicas de desarrollo rural impulsadas desde hace varias décadas en el territorio nacional. Es evidente que en general se ha tratado de una serie de propósitos discursivos, por cuanto no fueron diseñadas ni definidas desde los ámbitos locales, prueba de ello son las transformaciones territoriales como así también los contrastes sociales y económicos que se han producido tanto en ámbitos rurales naciones como provinciales.

En este trabajo se intenta poner de manifiesto la relación existente entre desigualdad, territorio y desarrollo, en el marco de las políticas de desarrollo rural territorial dirigidas teóricamente a beneficiar a familias de escasos recursos y vinculadas con acciones que surgen desde los actores locales. Para ello nos centramos en la región del centro y sudoeste chaqueño, donde el cultivo de algodón, principal actividad de miles de pequeños productores, entra en disputa por el territorio, como consecuencia de la alta rentabilidad que adquiere el cultivo de la soja transgénica. Ello pone en evidencia las transformaciones socio económico y territorial que se dan como consecuencia del avance de la frontera agropecuaria en el marco de las implicancias de la globalización en ámbitos locales.

Publicado en formato digital: Mgter. Prof. Viviana C. PERTILE. DESARROLLO RURAL TERRITORIAL Y TRANSFORMACIONES TERRITORIALES. Proyectos epara las igualdades o desigualdades? . Resúmenes. Revista Geográfica Digital. IGUNNE. Facultad de Humanidades. UNNE. Año 11. No 22. Julio - Diciembre 2014. ISSN 1668-5180. Resistencia, Chaco. En: http://hum.unne.edu.ar/revistas/geoweb/default.htm 


\title{
La cuestión rural en la Argentina
}

lleva consigo una diseusión intensa, prolongada y sin ressolver

\author{
$\Downarrow$ \\ desigualdad y al despoblamiento \\ zonas rurales
}

\author{
$\checkmark$ \\ pora effertividad que tuvieron las políticas \\ públizas de destarrollo rural impulsades desde \\ hace varias décadas en el territorio nacional.
}




\section{Desarrulla - Territariu}

san realidades

\section{san conceptas}

sun políticas pudemas ver

D

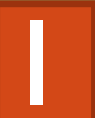


un término, es un concepto, una realidad y una definición política

lo usamos como cualquier palabra que define algo

se lo vinculan con la Econ.se lo asocia con el progreso desarrollo también puede estar asociado con algo que ya aconteció y con el futuro

\section{entonces}
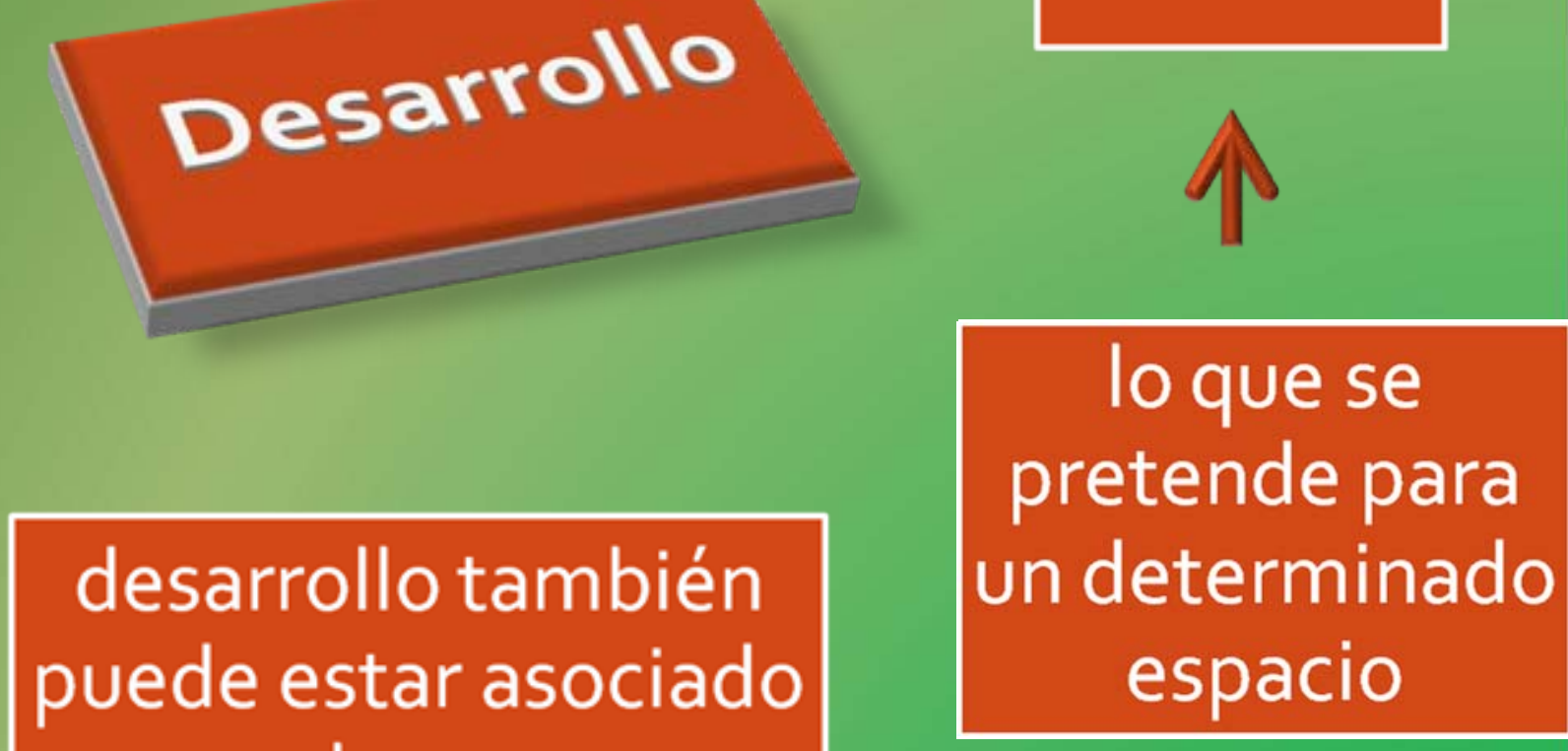


\section{Desarrollo}

comienza en la historia latinoamericana en el período de posguerra

Desde ese momento y a partir de los años 60 propuestas

Gobiernos desarrollista
Desarrollo /ligado al Territorio

Desarrollo Nacional

\section{Programas, Planes y Organismos}

Consejo Federal de Inversiones /CFI

orientadoa la sustentab.de procesos de desarrollo regional
Consejo Nacional de Desarrollo/CONADE organismo esencialmente consultivo y técnico-P/fijar obj. de desarrolllo 


\section{A partir /aplicación de planes y programas}

Desarrollo en forma desigual- centro y resto

regional

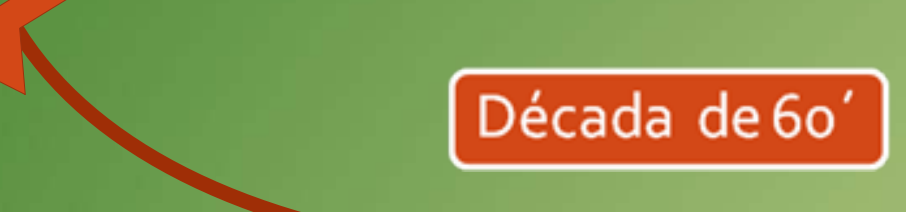

D. Local, D. Territorial, D. Ambiental, D.Sustentable, D. R. Territorial

$\sqrt{2}$

Pol. de Desarrollo/superar las diferencias

Manzanal - detrás de todas las concepciones e ideas de DESARROLLO hay EXPRESIONES de PODER 


\section{Desarrrollo y}

\section{Si detrás de la idpa de desarrollo se

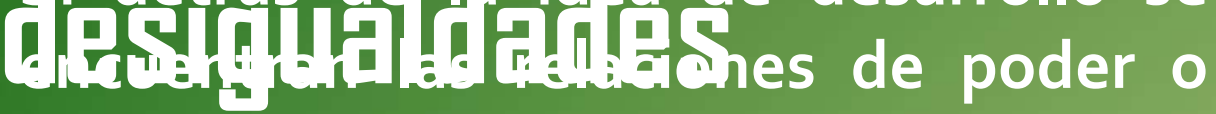 disputas de poder}
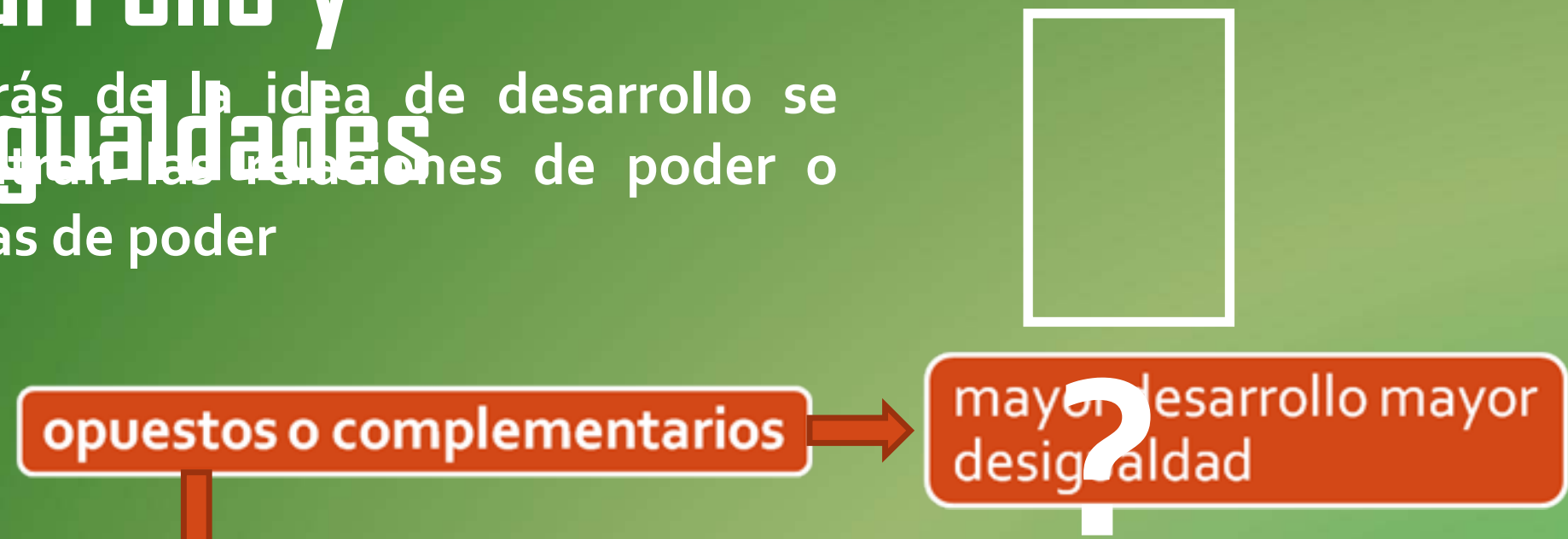

si se genera más desarrollo o aumenta el desarrollo, disminuyen las desigualdades

en ningún plan o programa /el objetivo es producir más desarrollo para lograr $>$ concentración- la realidad-según informes de Cepal/cada vez >desigualdad

desde esta mirada $\Rightarrow$ Es Complementario

Hay otra realidad, otros intereses en juego, tendientesa un desarrollo concentrado y a favor de la extranjerización

El territorio del Centro y Sudoeste Chaqueño 


\section{Cultivo de Soja - Superficie Sembrada.}

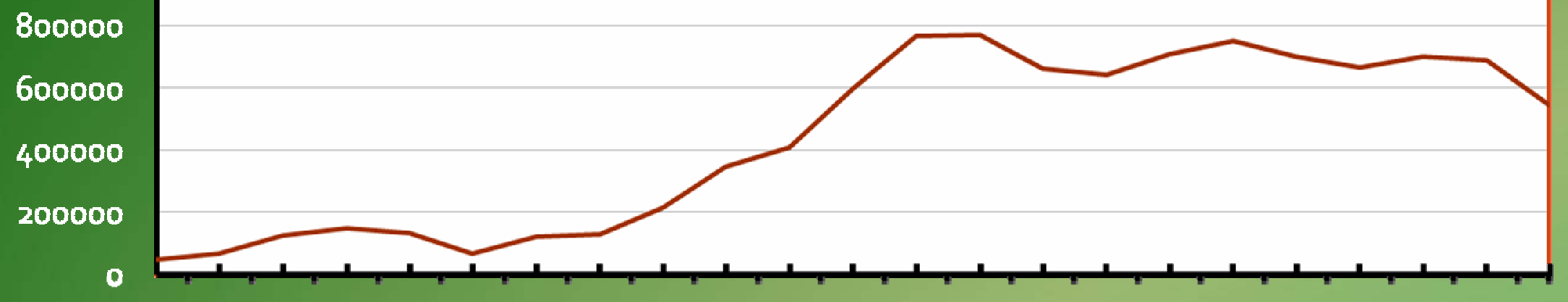

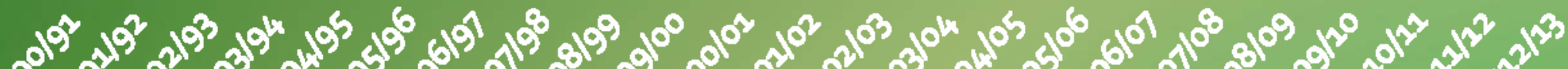

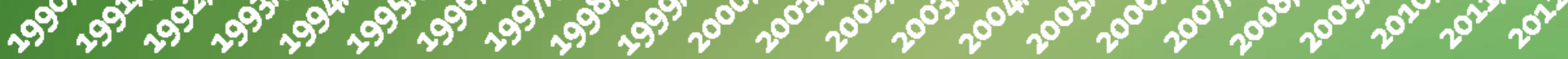
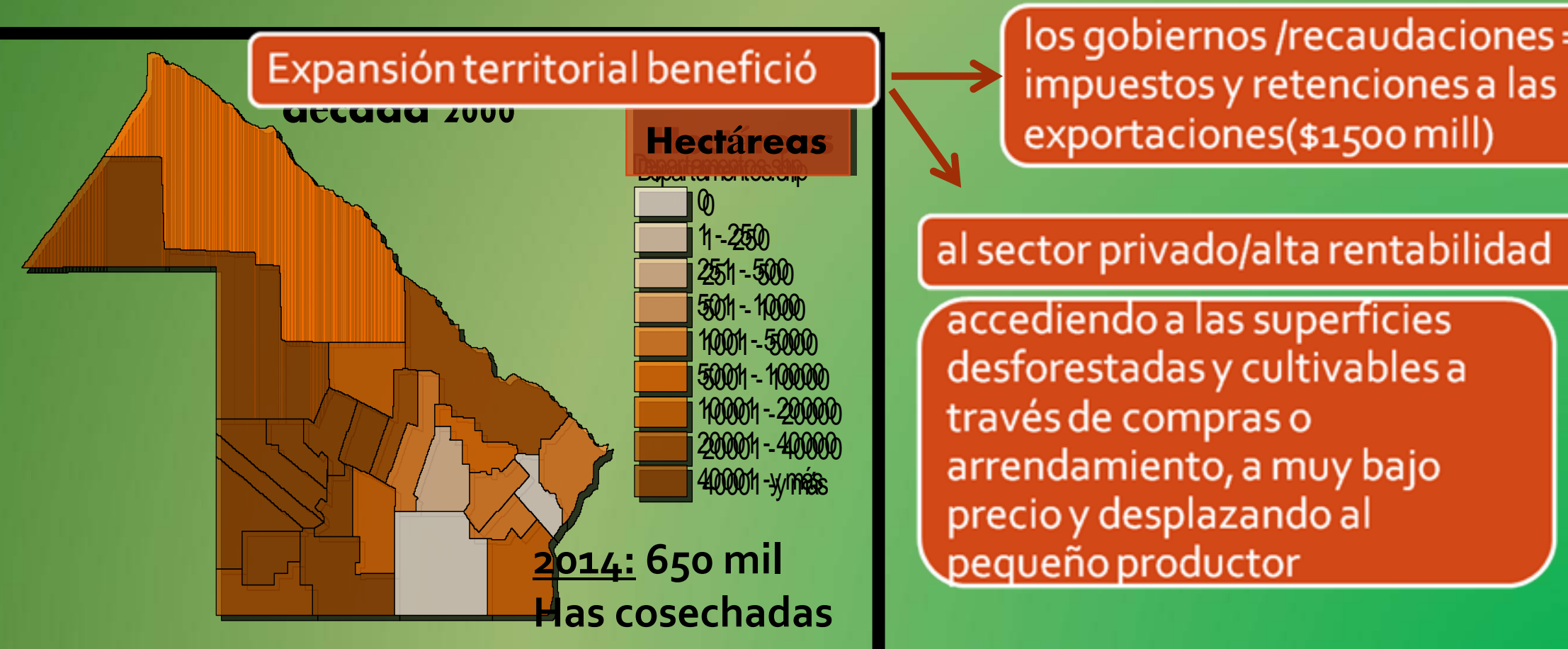


\section{Territorio / vineulación ton dastarrollı}

\section{Complejizando}

\section{Va mas allá de lo que entendemos como soberanía de un Estado}

Schejtman y Berdegué

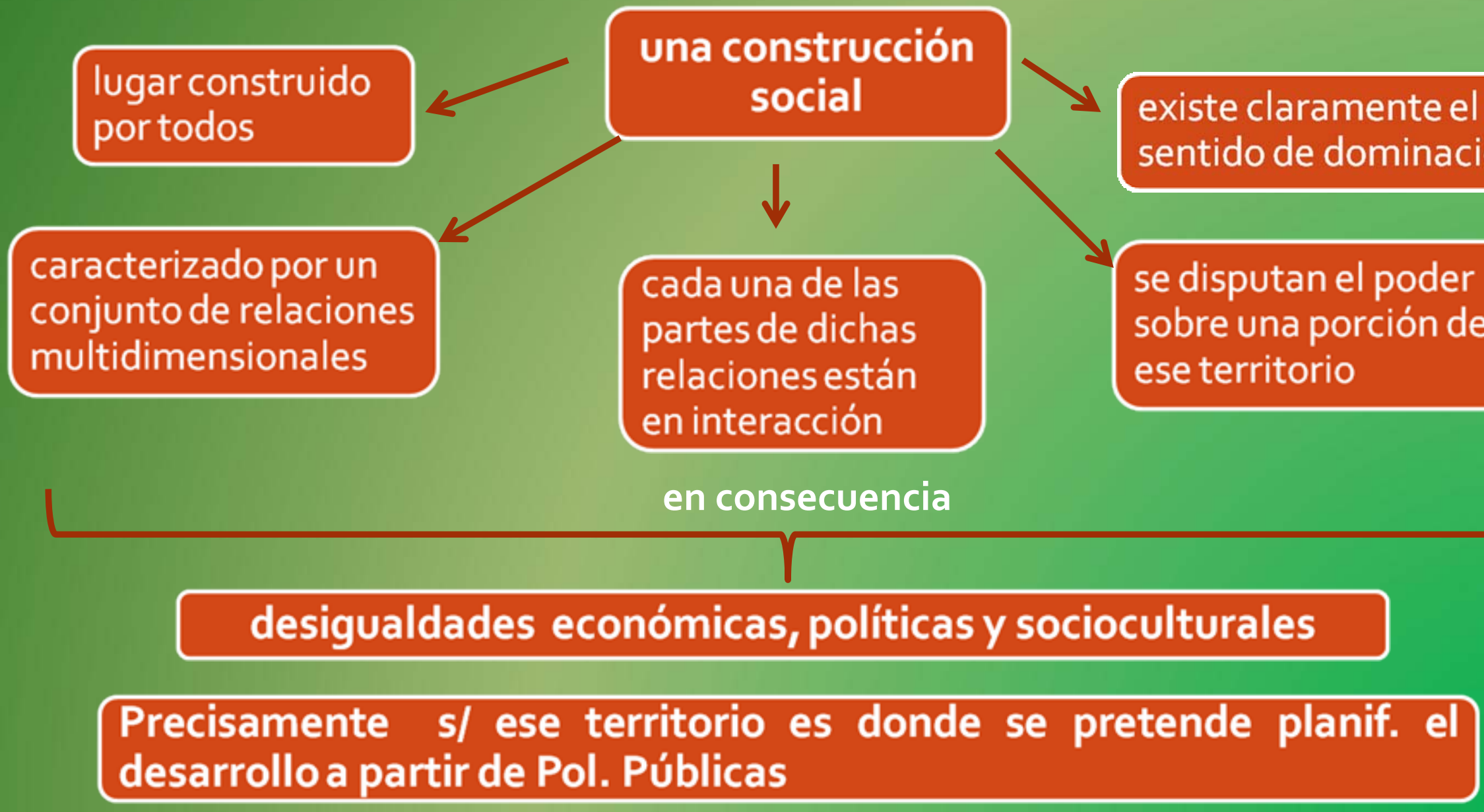




\section{Las políticas públinas y al dessarrulla territarial rural}

Los Programas de Desarrollo Rural
Constituyen políticas públicas de mas de 20 años

\section{Objetivo}

Mejorar las condiciones de vida, aumentar los ingresos de los PP tratando de reducir los efectos frente a las políticas de ajuste y desregulacióny complementariamente, modernizar, reconvertiry diversificar las explotaciones para al alcanzar niveles de productividad sustentables y con capacidad competitiva

\section{desde distintos organismos:}

SAGPYA

PSA (Programa Social Agropecuario), PROINDER (Proyecto de Desarrollo de Pequeños Productores Agropecuarios), PRODERNEA (Programa de Desarrollo Rural de las Provincias del Noreste Argentino)

INTA

PROFEDER (Programa Federal de Apoyo al Desarrollo Rural Sustentable, Cambio Rural (Programa Federal de Reconversión Productiva para la Pequeña y Mediana Empresa Agropecuaria, Pro-Huerta 


\section{PRDDERNEA}

Se inicia en el año 1999 y finaliza en el año 2007

$\|$

propósito $\rightarrow$

contribuir a superar las condiciones que generan la pobreza rural a través del aumento sostenible del ingreso y de la capacidad de autogestión de pobladores rurales e indígenas

\section{Resultadus pasitivas \\ Debilidades}

-Contribuyeron a que la cuestión campesina se hiciera visible.

-Permitieron generar formas asociativas y actitudes cooperativas.

-se Integró a los beneficiarios directos en la estructura de gestión de los programas (un represent. p/c regióngenerando capital social en algunos ámbitos locales a favor de las familias beneficiarias de pequeños productores)

-Avanzaron con mayor formalidad (en facturación, jubilación, seguro social).
- Logros acotados a un número de beneficiarios limitado dentro de los pobres rurales.

-Falta de evaluaciones que analicen la correspondencia entre lo planeado y lo ejecutado.

-Falta de conexión entre los programas / superposición de programas/se disputaban beneficiarios

•Discontinuidad en el financiamiento.

-Escasa difusión de resultados y estudios/no se conocía cómo se trabajaba - cómo se evaluaba 


\section{moda de}

\section{tierre}

- Es indiscutible la demostración de poder que ejercen ciertos sectores privilegiados al señalar qué debemos hacer para desarrollarnos.

- La permanente propuesta de alternativas para lograr el desarrollo rural se ha convertido es un instrumento del poder dominante para plantear ilusiones que mantengan a la población rural de menores recursos imaginando un futuro más ventajosos y superador.

- Tanto los gobiernos de turno como los sectores económicos de poder consideran al desarrollo como sinónimo de crecimiento económico en contraposición a la desigualdad social que dicho proceso genera. 


\section{Bibliografia}

BOZZANO, Horacio (2009): Territorios posibles. Procesos, lugares y actores. Ed. Lumiere. Buenos Aires. Argentina.

DUBOIS, Alfonso (2006): Un Concepto de Desarrollo Para el Siglo XXI. En: Revista Asuntos: Revista Asuntos Económicos y Administrativos. Universidad de Manizales. Facultad de Economía y Administración de Empresas.

ESTEVA, Gustavo, 1996. Desarrollo. En SACHS, W. (editor) Diccionario del desarrollo. Una guía del conocimiento como poder. Perú: PRATEC, 1996. 383p. p. 52-79.

BLANCO, Jorge (2007). "Espacio y territorio: elementos teórico conceptuales implicados en el análisis geográfico". En María Victoria Fernández y Raquel Gurevich (coords.), Geografía: nuevos temas, nuevas preguntas. Un temario para su enseñanza (pp. 37-64). Buenos Aires: Editorial Biblos.

FERNANDES, Bernardo Mançano (2010). Territorios en disputa: campesinos y agrobusiness. en :

GONZÁLEZ DE CANALES LÓPEZ-OBRERO, F. (2003). Concepto de territorio. Nota técnica del Instituto de Desarrollo Comunitario. Fundación INFODAL. Madrid. En: Martínez de Anguita, Pablo (2008):

INTA 2014: Programa Federal de Apoyo al Desarrollo Rural http://inta.gob.ar/documentos/profeder-programa-federal

Keynes, John Maynard, 2005: Teoría general de la ocupación, el interés y el dinero. Ed. Fondo de Cultura Económica. Bs. As. Argentina

LAGUNA MARÍN-YASELI, María (2007): Más de 20 años de políticas de desarrollo rural: la experiencia de las políticas regionales en el Pirineo aragonés. Editorial: Universidad de Zaragoza. España

MANZANAL y SNEIDER (2011) Agricultura Familiar y Políticas de Desarrollo Rural en Argentina y Brasil (análisis comparativo, 1990-2010). Revista Interdisciplinaria de Estudios Agrarios N 34, p. 35-71, CIEA, FCE, UBA, ISSN 1514-1535, 1er semestre 2011, Buenos Aires.

MANZANAL, Mabel (2011) Desarrollo, territorio y desigualdad en la globalización. Conflictos actuales en la agricultura familiar del nordeste de Misiones, Argentina. En: Revista Mundo Agrario, vol. 12, n 23, segundo semestre de 2011. Universidad Nacional de La Plata. Facultad de Humanidades y Ciencias de la Educación. Centro de Historia Argentina y Americana.

MANZANAL, Mabel (2013). "Poder y desarrollo. Dilemas y desafíos frente a un futuro ¿cada vez más desigual?". En Manzanal, M. y Ponce, M. (2013) La desigualdad ¿del desarrollo?. Controversias y disyuntivas del desarrollo rural en el norte argentino. Ediciones CICCUS, Buenos Aires.

MARYVONNE Le Berre, (1992). Concepto de territorio. En Hypergeo, Enciclopedia electrónica http://www.hypergeo.eu/spip.php?article514

MINISTERIO DE AGRICULTURA, GANADERIA Y PESCA: Primer Encuentro Regional de PRODERNEA En: http://64.76.123.202/new/oo/programas/prodernea/publicaciones/publicaciones.php?carpeta=Desarrollo_Rural

MINISTERIO DE ECONOMÍA Y PRODUCCIÓN (2003): Los programas de desarrollo rural ejecutados en el ámbito de la SAGPYA. Serie estudios e investigaciones. Buenos Aires

NEIRA ALVA, Eduardo (1974). "Las políticas de desarrollo regional en América Latina", Planificación regional y urbana en América latina, ILPES-ILDES, Siglo XXI, México. Citado en

PERTILE, V. y TORRE GERALDI, A (2009): "Cambios productivos en el sector agrícola de la provincia del Chaco: la sojización y sus consecuencias socioeconómicas en los pequeños productores rurales a fines del siglo XX". En: MORELLO, Jorge y Andrea F. RODRÍGUEZ (editores) El Chaco sin Bosques: La Pampa o el Desierto del futuro GEPAMAUniversidad de Buenos Aires -UNESCO. Editorial Orientación Grafica

PROINDER /DIRECCIÓN DE DESARROLLO AGROPECUARIO (2007): Los pequeños productores en la República Argentina

REPETTO, Fabián. (1998). Notas para el análisis de la política social: Una propuesta desde el institucionalismo. EN Perfiles Latinoamericanos. FLACSO. Año 7 № 12 SANTOS, Milton (1986). Por uma geografia nova. Da crítica da Geografia a uma Geografia Crítica. Terceira edicao. ED. HUCITEC, São Paulo, Brasil.

SCHEJTMAN, Alexander y BERDEGUÉ, Julio (2004). Desarrollo territorial rural. Debates y temas rurales No 1. Rimisp / Centro Latinoamericano para el desarrollo rural, Santiago de Chile. Disponible en

SCHERJTMAN, Alejandro y BARSKY, Osvaldo (2008): El desarrollo rural en la Argentina. Ed. Siglo XXI. Buenos Aires, Argentina

SECRETARIA DE AGRICULTURA, GANADERIA Y PESCA, (1997): Estrategia de Desarrollo Rural Hacia el Siglo XXI. SAGPyA. Buenos Aires.

TAMAYO SAENZ, Manuel (1997).El análisis de las políticas Públicas. En: Bañón, Rafael y Carrillo, Ernesto (compiladores). La Nueva Administración Pública, Alianza Universidad, Madrid 